\title{
Holding the baby: head downwards positioning for physiotherapy does not cause gastro-oesophageal reflux
}

\author{
G.E. Phillips*, S.E. Pike*, M. Rosenthal**, A. Bush**
}

Holding the baby: head downwards positioning for physiotherapy does not cause gastrooesophageal reflux. G.E. Phillips, S.E. Pike, M. Rosenthal, A. Bush. CERS Journals Ltd 1998.

ABSTRACT: The head-downwards tipped position for physiotherapy has been claimed to exacerbate gastro-oesophageal reflux (GOR) in infants with cystic fibrosis (CF). This was investigated using lower oesophageal $\mathrm{pH}$ monitoring during physiotherapy.

Twenty-one infants (age range 1-27 months) with respiratory disorders $(\mathrm{CF}=11)$, undergoing lower oesophageal $\mathrm{pH}$ monitoring were recruited. Subjects received two physiotherapy episodes in random order, $\mathrm{A} / \mathrm{B}$ or $\mathrm{B} / \mathrm{A}, 12 \mathrm{~h}$ apart. A began the gravityassisted positioning head downward tip for: right lower lobe, middle lobe, left lower lobe and lingula; then supine with no tip for anterior segments of the upper lobes followed by apical segments of upper lobes in a sitting position. $B$ was in the reverse order. Intermittent chest clapping was carried out for $4 \mathrm{~min}$ in each position by a physiotherapist blinded to the $\mathrm{pH}$ data.

During episode $\mathrm{A}$, the median change in $\mathrm{pH}$ from baseline was -0.32 (range -2.07 to +1.0 ) in non-CF subjects (Ns) and $\mathbf{- 0 . 5 2}$ (range -2.7 to +0.52$)$ in $\mathrm{CF}$ subjects $(\mathrm{p}<0.02)$. During episode $B$, the median change in non-CF subjects was -0.1 (Ns; range -1.7 to $\mathbf{- 0 . 1 5}$ ) and in CF subjects was $\mathbf{- 0 . 0 5}$ (Ns; range $\mathbf{- 0 . 6 7}$ to +0.5 ). There was no order effect for positioning. In the CF subjects the sitting position was twice as likely to have the lowest $\mathrm{pH}$ measurement during physiotherapy than the other positions $(\mathbf{p}<0.04)$.

In conclusion, the head-downward tipped positioning for physiotherapy treatment neither induces nor aggravates gastro-oesophageal reflux. There is no justification for routinely changing the way in which infant physiotherapy is carried out.

Eur Respir J 1998; 12: 954-957.
Depts of *Physiotherapy and **Paediatric Respiratory Medicine, Royal Brompton Hospital, London, UK.

Correspondence: G. Phillips

King's College London

Campden Hill Road

London

W8 7AH

UK

Fax: 441713334032

Keywords: Cystic fibrosis gastro-oesophageal reflux physiotherapy

Received: October 11997

Accepted after revision June 201998
In infants with respiratory disorders complicated by excess bronchopulmonary secretions and sputum retention, chest physiotherapy to enhance airway clearance is an essential part of management. Established clinical practice consists of gravity-assisted positioning combined with periods of chest clapping. It is possible that gastro-oesophageal reflux (GOR) may cause or complicate recurrent respiratory problems [1], but whether or not head-downwards positioning aggravates GOR has not been determined. A recent study claimed that modified head-up positioning for physiotherapy caused less reflux than treatment in tipped positions [2] but this has been disputed [3]. In three previous studies reporting GOR as a consequence of chest physiotherapy, it is unclear whether the headdownward tipped positions were investigated, but the physiotherapy described would not be considered orthodox since it also consisted of the use of abdominal thrusts, routine tracheal rubs and nasopharyngeal suction [4-6]. This study was, therefore, designed to determine whether established current physiotherapy practice for infants causes or exacerbates GOR.

\section{Methods}

\section{Subjects}

Twenty-one infants (six male) were recruited, with a median age of 8 months (range 1-27 months), undergoing
$20 \mathrm{~h}$ of lower oesophageal $\mathrm{pH}(\mathrm{LOpH})$ monitoring to evaluate possible GOR. Eleven infants had cystic fibrosis (CF). The remainder were being investigated for either or both of recurrent wheeze and recurrent lower respiratory tract infection. Infants $<2.5 \mathrm{yrs}$ of age, requiring chest physiotherapy, were eligible for entry into the study. Exclusion criteria were: a nasogastric tube in situ, receiving systemically administered $\beta$-agonists [1] or a coexisting medical condition which contra-indicated being placed in a headdownwards tipped position, e.g. congenital heart disease. The study protocol was approved by the Ethics Committee and informed, written consent was obtained from all parents or carers.

\section{pH measurements}

LOpH measurements were made using Synectics $\mathrm{pH}$ monitoring equipment (Digitrapper Mark III ambulatory system; Synectics Medical, Stockholm, Sweden). A twopoint calibration was performed on each antimony electrode before use. The location of the electrode was determined in accordance with the protocol of the European Society of Pediatric Gastroenterology and Nutrition [7]. Foodthickening agents were not used for feeds and subjects were not receiving antireflux medication. 


\section{Physiotherapy}

Each subject received two physiotherapy treatment sessions from an experienced physiotherapist during their LOpH monitoring. Chest clapping with pauses was carried out for $4 \mathrm{~min}$ in six gravity-assisted positions. These positions were: left side lying with $20^{\circ}$ head-downward tip for the right lower lobe; left side lying with a quarter turn to supine and $15^{\circ}$ downward tip for the middle lobe; right side lying and $20^{\circ}$ tip for the left lower lobe; right side lying with a quarter turn to supine and $15^{\circ}$ tip for the lingula; and supine lying, no tip for the anterior segments of the upper lobes and upright sitting for the apical segments of the upper lobes. These positions constituted treatment order A, with treatment order B the reverse. From an envelope, subjects were randomized to receive physiotherapy A/B or B/A. Treatments were carried out at least $2 \mathrm{~h}$ postprandial and $12 \mathrm{~h}$ apart, one in the evening and one in the morning. $\mathrm{pH}$ recordings were made at one-minute intervals during a four-minute basal run-in period and throughout treatment by an independent observer. The physiotherapist performing the treatment was blind to the $\mathrm{pH}$ data. Data were examined using $\mathrm{pH}$ software from Gastrosoft (Synectics Medical). Acid reflux was defined as a fall in $\mathrm{LOpH}$ to $<4$ for at least $15 \mathrm{~s}$. The percentage of total time where $\mathrm{pH}<4$ is the reflux index (RI) [7].

\section{Analysis}

Data were analysed using SPSS for Windows v. 7.0 (SPSS, Chicago, IL, USA), with a significance level of $\mathrm{p}<0.05$. Analysis of variance (ANOVA) was used to determine the effects of treatment order and between-subject variability. The median baseline value for each subject was compared with the lowest $\mathrm{pH}$ measurement occurring during physiotherapy for each episode, and for each physiotherapy position. The standard deviation for the group having been determined, a retrospective calculation was made of the power of the study to detect a significant change.

\section{Results}

The $20 \mathrm{~h} \mathrm{LOpH}$ results demonstrated a greater overall incidence of GOR in the CF subjects $(\mathrm{p}<0.02)$, with $8 / 11$ having RI >3.4\% (range 1-21\%) compared with $3 / 10$ non$\mathrm{CF}$ (range $1-16.5 \%$ ).

During physiotherapy, the median change in $\mathrm{pH}$ from baseline to the lowest $\mathrm{pH}$ value occurring in treatment episode A was -0.32 (range -2.07 to +1.0 ) in non-CF subjects $(\mathrm{p}=$ nonsignificant $(\mathrm{NS})$ ) and -0.52 (range -2.7 to +0.52 ) in $\mathrm{CF}$ subjects $(\mathrm{p}<0.02)$. The median change in $\mathrm{pH}$ from baseline to the lowest $\mathrm{pH}$ value during episode $\mathrm{B}$ was -0.1 (range -1.7 to -0.15 ) in non-CF subjects (ns) and -0.05 (range -0.67 to +0.5 ) in $\mathrm{CF}$ subjects (Ns). In the CF subjects, there was no significant difference in the $\mathrm{pH}$ changes during episodes A and B. ANOVA demonstrated no difference in $\mathrm{pH}$ measurements related to order of positioning (A/B or B/A). Between-subject variability was the most potent factor influencing $\mathrm{pH}$ changes $(\mathrm{p}<0.001)$.

Figure 1 shows individual patient $\mathrm{pH}$ median and range measurements for both $\mathrm{CF}$ and non-CF subjects during physiotherapy. For subject number 6 , the only non-CF

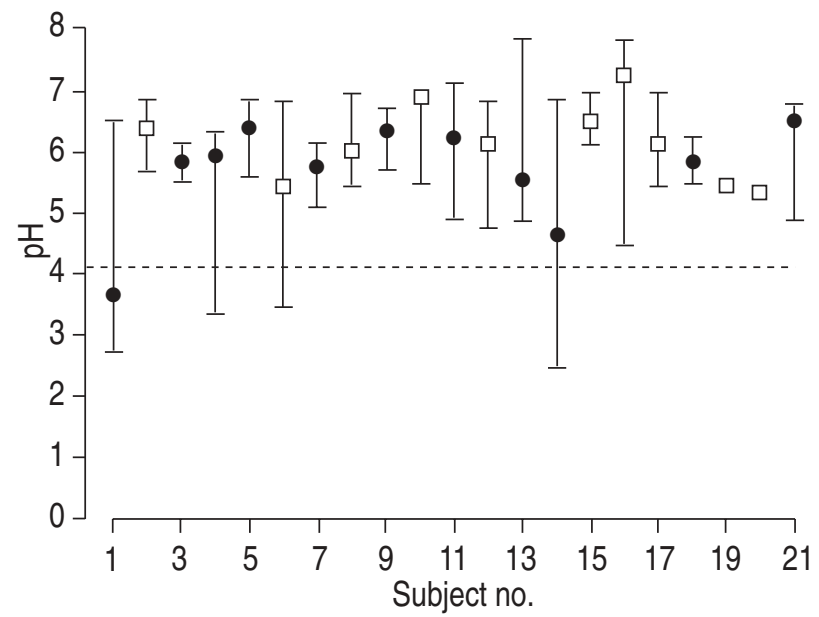

Fig. 1. - Individual patient $\mathrm{pH}$ measurements during physiotherapy. cystic fibrosis (CF); $\square$ : non-CF patients; - - - - : reflux threshold. Values are shown as median and range.

subject to experience a $\mathrm{pH}<4$ with physiotherapy, the fall in $\mathrm{pH}$ occurred during both episodes whilst being treated in the upright sitting position but at no other time. In subjects 1 and 4 (both $\mathrm{CF}$ ), reflux occurred $(\mathrm{pH}<4)$ during treatment episode A when they were placed in the sitting position. With treatment episode $\mathrm{B}$, both subjects were refluxing during baseline recordings and subject 1 continued to reflux throughout physiotherapy. Subject 4 , however, stopped refluxing when positioned tipped head downwards for treatment. The third patient with $\mathrm{CF}$ with a fall in $\mathrm{pH}<4$ throughout baseline and episode A (subject 14) demonstrated no $\mathrm{pH}<4$ during episode $\mathrm{B}$. In $\mathrm{CF}$ subjects, the sitting position was twice as likely to have the lowest $\mathrm{pH}$ during physiotherapy than predicted (Chisquared=4.27; $\mathrm{p}<0.04$; fig. 2 ).

The standard deviation for $\mathrm{pH}$ for the group was $0.7 \mathrm{pH}$ units. Studying 11 children with $\mathrm{CF}$ gives a $55 \%$ chance of detecting a change of $0.5 \mathrm{pH}$ units at the $5 \%$ level and a

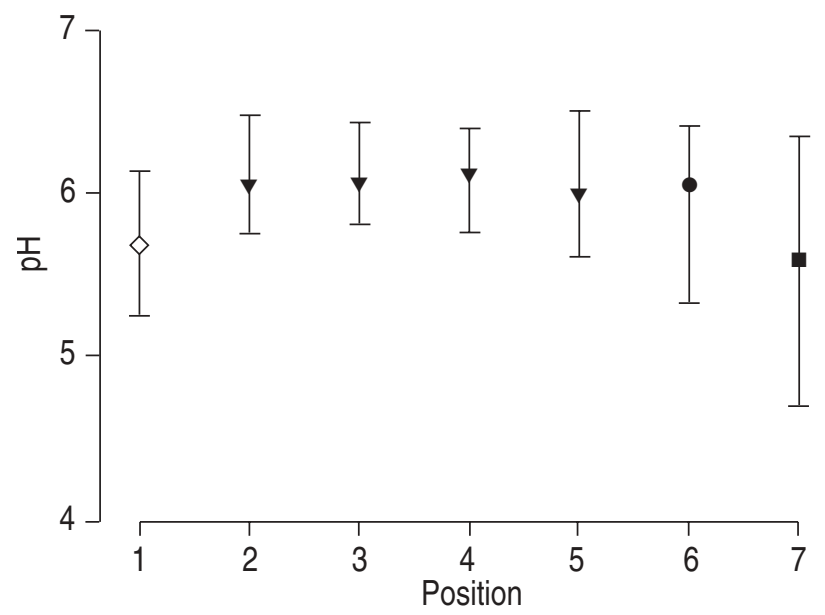

Fig. 2. - $\mathrm{pH}$ in cystic fibrosis subjects versus physiotherapy position. Position 1: baseline recordings $(\diamond)$; positions 2-5: subjects positioned with a head-downward tip $(\boldsymbol{\nabla})\left(2\right.$ : left side lying with $20^{\circ}$ head-downward tip; 3: left side lying with $1 / 4$ right turn to supine and $15^{\circ}$ tip; 4 : right side lying with $20^{\circ}$ head-downward tip; 5: right side lying with $1 / 4$ right turn to supine and $15^{\circ}$ tip); position 6 : supine lying $(\bullet)$; and position 7: upright sitting (ם). 
$70 \%$ chance of detecting a change between positions. Studying 21 patients (the whole group) gives at least an $80 \%$ chance of detecting differences between positions at the $5 \%$ level.

\section{Discussion}

The overall $\mathrm{LOpH}$ monitoring results show that the incidence of GOR in CF was more common than in the other respiratory non-CF conditions investigated. An incidence of $73 \%$ was found, which appears to be in line with the $81 \%$ described by MALFOO and DAB [8]. This study demonstrates, however, that performing chest physiotherapy in head-downward tipped positions neither caused nor aggravated GOR. The lowest $\mathrm{pH}$ measurements re-corded during physiotherapy tended to occur when sitting. Nevertheless, an actual drop to a $\mathrm{pH}$ of $<4$ occurred only rarely; in two CF subjects, when sitting, during one treatment episode and in one non-CF subject, when sitting, during both treatment episodes. The sitting position for physiotherapy is discontinued once the infant is walking.

The sitting position is generally considered that in which GOR is worst in infants $[9,10]$. A slumped position raises intra-abdominal pressure and this is one mechanism that may induce GOR [11]. Transient relaxations of the lower oesophageal sphincter (LOS), a cause of GOR, especially in CF [12], are also more frequent when sitting [13]. A head-downward tipped position may prevent GOR as intra-abdominal pressure falls and intrathoracic pressure rises, thus actually reducing the adverse gradient across the LOS which would otherwise favour reflux [14]. Furthermore, diaphragmatic work is increased in the tipped position, which would also enhance LOS competence [15]. In the head-downward positions, gastric contents would distend and lie in the areas of the stomach curvatures. TOBIN et al. [16] found that GOR was decreased in the left lateral position and speculated that with the greater curvature acting as a reservoir, the pressure of gastric contents against the LOS would be reduced.

One limitation of the present study was the inability to identify alkaline reflux. Changes in hydrogen ions at concentrations over $\mathrm{pH} 7$ are too small to be sensed by $\mathrm{pH}$ monitoring [17]. The importance of alkaline reflux in GOR disease is, however, controversial and clinical decisions are usually based on the presence or absence of acid reflux. This study could be criticized because neither aspiration nor respiratory symptoms induced via neural reflex arcs [18] were examined. Detection of aspiration would have necessitated isotope studies which were thought to be neither practical nor ethical. The cause and effect association between GOR and lung disease is also controversial [19].

The definition of a pathological RI being $>3.4 \%$ is arbitrary and may be considered by some to be a little too low. There is no agreed, defined cut-off value for normal and abnormal reflux in the published literature. VANDENPLAS et al. [20] described $10 \%$ as the 95 percentile, with $4 \%$ as the 50 percentile. The $\mathrm{pH}$ software from Gastrosoft defines abnormal RI as $>3.4 \%$. In clinical practice we use a figure of $3.4 \%$ in our assessment of the presence or absence of GOR as frequently patients with this RI, even at borderline, have symptomatic reflux warranting medical management. An increase in RI to the 5\% figure most recently proposed [16] does not change the incidence of pathological reflux presented in our study. Furthermore, applying the present definition to the data presented by MALFOOT and $D_{A B}$ [8] does not alter their incidence of pathological GOR. Thus, we consider that our conclusions are not affected by the definition of pathological GOR used.

It is acknowledged that this study had a relatively small number of subjects and that in some infants it is possible that the head-downward tipped positions may worsen gastro-oesophageal reflux. Therefore, individual evaluation of physiotherapy is recommended for infants undergoing lower oesophageal $\mathrm{pH}$ monitoring in whom clearance of excess bronchial secretions is indicated. The general advice not to tip infants for physiotherapy because of fears of worsening gastro-oesophageal reflux [2] is misplaced. There are no indications either to modify radically current clinical practice or to implement "routine" physiotherapy management of infants with cystic fibrosis.

\section{References}

1. Orenstein SP, Orentstein DM. Gastroesophageal reflux and respiratory disease in children. J Pediatr 1988; 112: 847-858.

2. Button BM, Heine RG, Catto-Smith AG, Phelan PD, Olinsky A. Postural drainage and gastro-oesophageal reflux in infants with cystic fibrosis. Arch Dis Child 1997; 76: $148-150$.

3. Taylor CJ, Threlfall D. Postural drainage techniques and gastro-oesophageal reflux in cystic fibrosis. Lancet 1997; 349: 1567-1568.

4. Demont B, Escourrou P, Vinçon Cl, Cambas H, Grisan A, Odièvre M. Effets de la kinésithérapie respiratoire et des aspirations naso-pharyngées sur le reflux gastro-oesophagien chez l'enfant de 0 à 1 an, avec et sans reflux pathologique. Arch Franç Pédiatr 1991; 48: 621-625.

5. Foster AC, Voyles JB, Murphy SA. Twenty four hour $\mathrm{pH}$ monitoring in children with cystic fibrosis: association of chest physical therapy to gastroesophageal reflux. Pediatr Res 1983; 17: Suppl. ii, 188A.

6. Vandenplas Y, Diericx A, Blecker U, Lanciers S, Deneyer $\mathrm{M}$. Esophageal $\mathrm{pH}$ monitoring data during chest physiotherapy. J Pediatr Gastroent Nutr 1991; 13: 23-26.

7. Working Group of the European Society of Pediatric Gastroenterology and Nutrition. A standardised protocol for the methodology of esophageal $\mathrm{pH}$ monitoring and interpretation of the data for the diagnosis of gastroesophageal reflux. J Pediatr Gastroent Nutr 1992; 14: 467-471.

8. Malfoot A, Dab I. New insights on gastro-oesophageal reflux in cystic fibrosis by longitudinal follow up. Arch Dis Child 1991; 66: 1339-1345.

9. Jolley SG, Johnson DG, Herbst JJ, Pena AR, Garnier RC. Assessment of gastroesophageal reflux in children by extended $\mathrm{pH}$ monitoring of the distal esophagus. Surgery 1978; 84: 16-23.

10. Orenstein SR, Whitington PF, Orenstein DM. The infant seat as a treatment for gastroesophageal reflux. $N$ Engl $J$ Med 1983; 309: 709-710.

11. Dodds WJ, Hogan WJ, Helm JF, Dent J. Pathogenesis of reflux esophagitis. Gastroenterology 1981; 81: 376-394.

12. Cucchiara S, Santamaria F, Andreotti MR, et al. Mechanisms of gastro-oesophageal reflux in cystic fibrosis. Arch Dis Child 1991; 66: 617-622.

13. Mittal RK, Holloway RH, Penagini R, Ashley Blackchaw L, Dent J. Transient lower esophageal sphincter relaxa- 
tion. Gastroenterology 1995; 109: 601-610.

14. Mansfield LE. Associations and interactions between esophagus and the lower respiratory tract. Pediatr Pulmonol 1995; 11: (Suppl.): 53-54.

15. Sindel BD, Maisels MJ, Ballantine TVN. Gastro-oesophageal reflux to the proximal oesophagus in infants with bronchopulmonary dysplasia. Am J Dis Child 1989; 143 : 1103-1106.

16. Tobin JM, McCloud P, Cameron DJS. Posture and gastro-oesophageal reflux: a case for left lateral positioning. Arch Dis Child 1997; 76: 254-258.

17. de Caestecker JS, Heading RC. Esophageal pH monitor- ing. Gastroent Clini N Am 1990; 19: 645-669.

18. Simpson H, Hampton F. Gastro-oesophageal reflux and the lung. Arch Dis Child 1991; 66: 277-279.

19. del Rosario JF, Orenstein SR. Evaluation and management of gastroesophageal reflux and pulmonary disease. Curr Opin Pediatr 1996; 8: 209-215.

20. Vandenplas Y, Goyvaerts H, Helven R, Sacre I. Gastroesophageal reflux, as measured by 24 hour $\mathrm{pH}$ monitoring, in 509 healthy infants screened for risk of sudden infant death syndrome. Pediatrics 1991; 88: 834840. 